$$
\begin{gathered}
N 69-32883 \\
C R-103671
\end{gathered}
$$

WHISTLER PROPAGATION IN A BOUNDED MAGNETOPLASMA

by

J. C. Lee

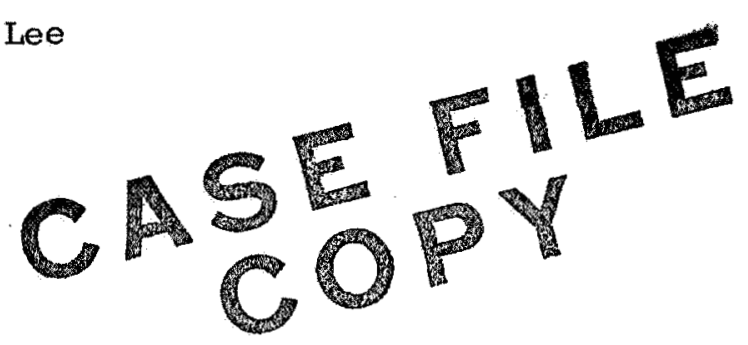

Technical Report

NASA Research Grant NGR 05-020-176

SU-IPR Report No. 318

May 1969

Institute for Plasma Research

Stanford University

Stanford, California 


\title{
WHISTLER PROPAGATION IN A BOUNDED MAGNETOPLASMA*
}

\author{
by \\ J. C. Lee \\ Institute for Plasma Research \\ Stanford University \\ Stanford, California
}

\begin{abstract}
For whistlers in bounded magnetoplasmas, a paradox has been pointed out by Wieder. His work shows that, below half the electron cyclotron frequency, the wave propagation characteristics do not approach those of plane wave solutions in unbounded magnetoplasma, even when the plasma density is increased so that the wavelength is arbitrarily small compared to the plasma transverse dimensions. In this paper, the apparent paradox is shown to result from the double reflection property of magnetoplasmas. Propagation characteristics in bounded plasma have been related to the unbounded case by considering plane waves doubly reflected at the plasma boundary. As a by-product, limitations for the validity of the quasistatic approximation for body waves in bounded magnetoplasmas have been established.
\end{abstract}

* This work was supported by the National Aeronautics and Space Administration. 
1. INTRODUCTION . . . . . . . . . . . . . . . . 1

2. DOUBLE REFLECTIONS . . . . . . . . . . . . . . . . 3

2.1 Whistlers Propagating at an Angle to the Magnetic Field . 3

2.2 Refractive Index Surfaces and Resonance Cones . . . . . 5

2.3 Generalized Snell's Law . . . . . . . . . . . 7

2.4 The Booker Quartic. . . . . . . . . . . . . . 10

2.5 The Reflected and Refracted Fields. . . . . . . . . 11

3. CALCULATION. . . . . . . . . . . . . . . . . 15

3.1 Explanation of Wieder's Paradox . . . . . . . . . 15

3.2 Limitations of the Quasistatic Approximation. . . . . . . 19

4. DISCUSSION . . . . . . . . . . . . . . . . 24

ACKNOWLEDGEMENTS. . . . . . . . . . . . . . . . . . . . 24

REFERENCES. . . . . . . . . . . . . . . . . . 25 


\section{ILLUSTRATIONS}

Figure

Page

1. CMA diagram and phase velocity surfaces . . . . . . . . . . 4

2. Whistler refractive index surfaces. . . . . . . . . . . . 6

3. Whistler resonance cone angle . . . . . . . . . . . . . . 8

4. Reflection and refraction of a plane wave at a sharp magnetoplasma boundary. . . . . . . . . . . . . . . . . . 9

5. Perpendicular and parallel wavenumbers in a bounded magnetoplasma $\left[\omega_{\mathrm{p}}^{2} / \omega^{2}=25, \theta_{\mathrm{I}}=10^{\circ}\right] . . . . . . . . . .16$

6. Principal and secondary reflection coefficients for whistler incident at an angle to a sharp magnetoplasma boundary $\left[\omega_{\mathrm{p}}^{2 / \omega^{2}}=25, \theta_{\mathrm{I}}=10^{\circ}\right] . . . . . . . . . .17$

7. Component plane waves in a magnetoplasma slab . . . . . . 18

8. Whistler dispersion characteristics in bounded magnetoplasma. 20

9. Comparison of body wave dispersion curves in magnetoplasma filled waveguide calculated from full Maxwell equations, and with quasistatic approximation (Gould 1961) . . . . . . 


\section{INTRODUCTION}

Slow right-hand polarized electromagnetic waves propagating in a magnetoplasma, parallel to the static magnetic field, are known in magnetospheric terminology as "whistlers." In the magnetosphere, where the plasma dimensions are very large compared with a whistler wavelength, and with the scale lengths of electron density and static magnetic field inhomogeneities, ray tracing techniques can be used to describe the propagation characteristics (Helliwell 1965). In a laboratory plasma, however, or in a field-aligned column of enhanced ionization in the magnetosphere, the effects of the plasma boundaries may be important in determining the propagation characteristics of these waves. It is this topic which forms the subject of the present paper.

A few boundary value problems for homogeneous plasma columns in a uniform axial magnetic field have been solved numerically, using the full Maxwel1 equations (Gould 1961, Formato and Gilardini 1962, Wieder 1964, Hedvall and Sjogren 1965. By comparing wave properties in bounded and unbounded magnetoplasmas, it has been found that the presence of sharp boundaries changes the wave dispersion drastically for certain ranges of parameters, sometimes beyond recognition from that prevailing in the unbounded case. For example, Wieder (1964) examined the properties of circularly symmetrical waves propagating in a dense, homogeneous, collisionless plasma, immersed in a uniform magnetic field and bounded by a conducting wall. He found that, below electron cyclotron resonance, an infinite number of propagating modes are possible. When the wavelength becomes much smaller than the transverse dimensions of the plasma, the dispersion characteristics of these waves approach those of the corresponding plane whistler waves only if the frequency is above about half the electron

cyclotron frequency. By intuition based on waveguide theory, however, one might expect the dispersion characteristics of the waves to approach those of the corresponding plane waves for all frequencies below the cyclotron frequency, in the limit of small wavelength. A way out of the difficulty was found by Hedvall and Sjogren (1965), who solved a boundary value problem for an infinite plasma slab numerically, including collisions. They showed that by making the collision frequency sufficiently large, 
the dispersion characteristics for waves below half the cyclotron frequency would approach those of plane whistler waves in an infinite plasma, provided the electron plasma frequency is adjusted so that the wavelengths are small compared to the plasma transverse dimensions. Although this avoids the paradox discovered by Wieder, it does not explain it. In what follows, our main purpose will be to do so.

Of the numerous papers concerned with waves propagating through bounded magnetoplasmas, the majority employ the quasistatic approximation (SmulIin and Chorney 1958, Trivelpiece and Gould 1959). In this approximation, the RF magnetic field is neglected $(\nabla \times \underset{\sim}{E} \approx 0)$, so that the RF electric field can be simply derived from a scalar potential. The quasistatic approximation greatly simplifies the numerical solution of the corresponding dispersion relations. The original justification for the quasistatic approximation, as described by Trivelpiece and Gould, was the slowness of the phase velocity, so that retardation effects can be neglected. This justification is not always satisfactory, since plane wave whistlers in unbounded plasmas are slow waves, yet they cannot be derived from quasistatic theory. On the other hand, phase velocities comparable to, or even greater than the velocity of light have been predicted by the quasistatic theory, which turned out to be very good approximations (Bevc 1966).

Since there should, in general, be a generic relationship between waves in bounded and unbounded media, the investigation of this relationship is important. In the work to be described, this relationship for whistlers in bounded and unbounded magnetoplasma is elucidated by consideration of double reflections at the boundaries. In particular, the paradox raised by Wieder is resolved, and the conditions for the validity of the quasistatic approximation are established. The plan of this paper is as follows: In Section 2, we will consider the double reflection at a general dielectric-plasma boundary for plane whistlers propagating at an angle to the magnetic field. In section 3, the computed results for the angles of reflection and reflection coefficients are given. They are used to explain Wieder's paradox, and to indicate the limitations of the quasistatic approximation in bounded magnetoplasmas. A general discussion of the results is given in Section 4 . 


\section{DOUBLE REFLECTIONS}

\subsection{Whistlers Propagating at an Angle to the Magnetic Field}

In a transversely bounded plasma, plane whistlers cannot propagate exactly along the boundary because of the circularly polarized nature of the wave. The dispersion relations for the two characteristic plane waves propagating in an arbitrary direction, in an infinite homogeneous cold plasma, in a uniform steady magnetic field, $B_{0}$, are given by the AppletonHartree equation (Budden 1961).

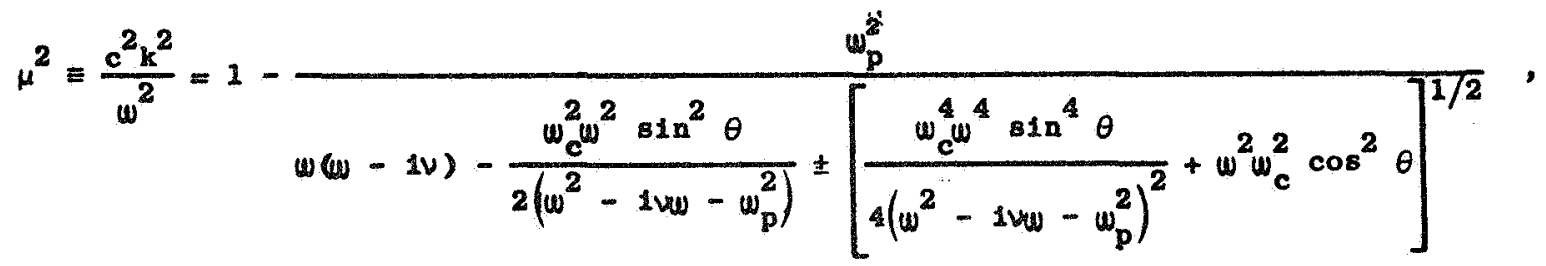

where $\mu$ is the refractive index; $c$ is the speed of light in free space; $\omega$ is the signal frequency; $k$ is the wavenumber; $\omega_{p}$ is the electron plasma frequency; $\omega_{c}$ is the electron cyclotron frequency; $v$ is the momentum transfer collision frequency, and $\theta$ is the angle between the direction of propagation and the static magnetic field, $\stackrel{\mathrm{B}}{0}_{0}$.

The dispersion relations of the different modes described by Eq. (1) can be conveniently characterized by their phase velocity surfaces, and a CMA diagram (Allis et al 1963), as shown in Fig. 1. Phase velocity surfaces are polar plots of the phase velocity in the plane containing $\underset{\sim}{\mathrm{B}}$. At fixed values of the parameters $\omega_{p}$ ' $\omega_{c}$, one obtains in general two nonintersecting phase velocity surfaces. These surfaces will change continuously as the parameters $\omega_{p}, \omega_{c}$ change, unless one passes through a point of resonance, $\mu \rightarrow \infty$; or point of cutoff, $\mu=0$. Region 8 of Fig. 1 is the region where the whistler is the only propagating mode.

When $\theta=0$, Eq. (1) reduces to

$$
\frac{c^{2} k^{2}}{\omega^{2}}=1+\frac{\omega_{p}^{2}}{\omega\left(\omega-i \nu \pm \omega_{c}\right)} .
$$




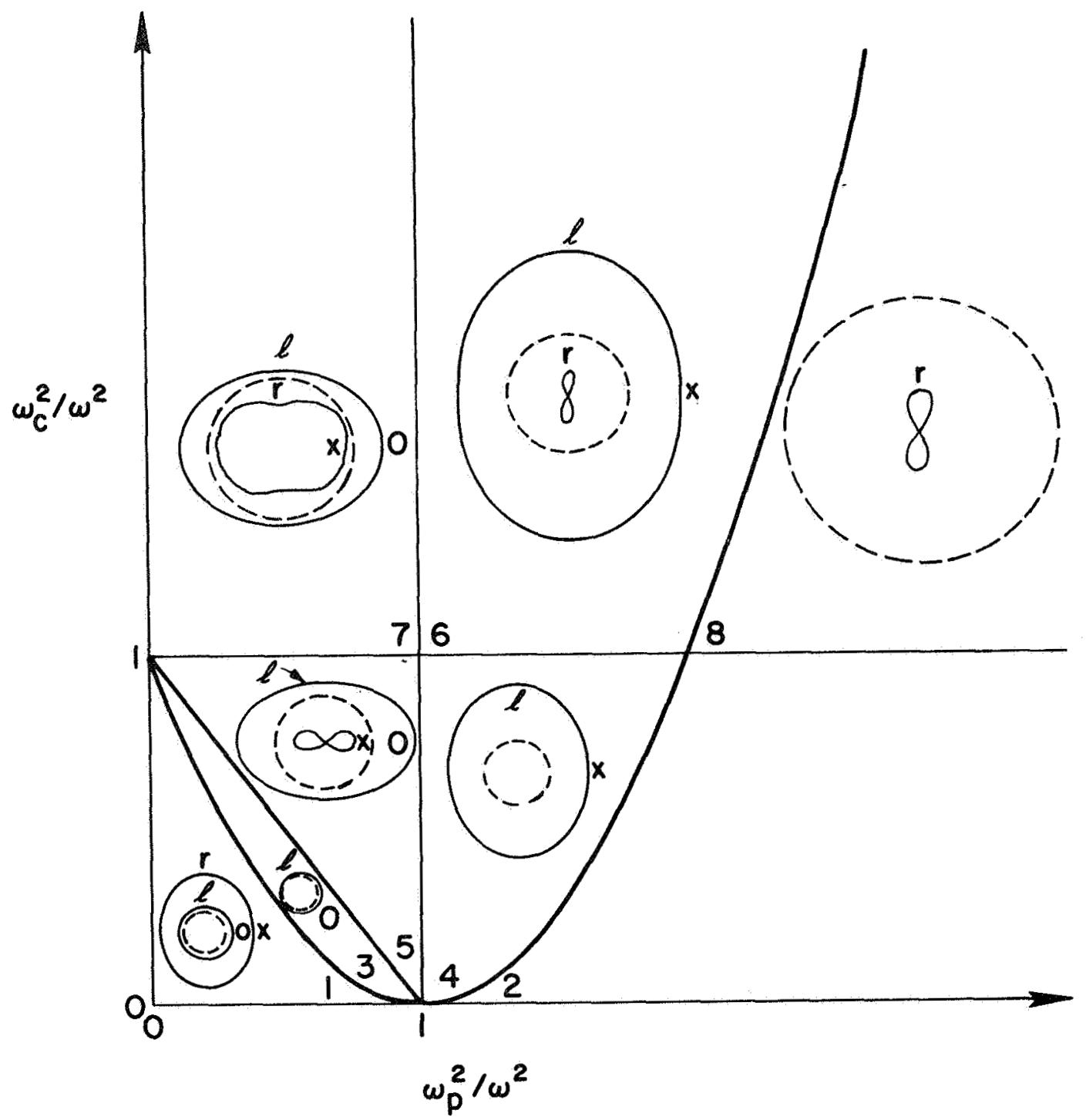

Fig. 1. CMA DIAGRAM AND PHASE VELOCITY SURFACES. 
Here the plus and minus signs before $\omega_{c}$ correspond to the left-and right-hand polarized waves. When

$$
\omega_{c}^{2} \omega^{2} \tan ^{2} \theta \sin ^{2} \theta \ll 4\left|\left(\omega^{2}-i v \omega-\omega_{p}^{2}\right)^{2}\right|
$$

Eq. (1) reduces to

$$
\frac{c^{2} k^{2}}{\omega^{2}}=1-\frac{\omega_{p}^{2}}{\omega\left(\omega-i v \pm \omega_{c} \cos \theta\right)}
$$

This is the well known "quasi-longitudinal" approximation (Budden 1961 ). For large $\omega_{p}^{2} / \omega^{2}$, the above approximation is good over a large range of $\theta$. When the condition of Eq. (3) is satisfied, the wave polarization is nearly circular, so that the approximation is sometimes called "quasicircular" (Allis et al, 1963).

\subsection{Refractive Index Surfaces and Resonance Cones}

By rotating the curve of refractive index vs. $\theta$ about the direction of the static magnetic field, the refractive index surface is generated. The polar plot of the refractive index in Fig. 2 shows sections of two distinct surfaces obtained for

$$
\frac{\omega}{\omega_{c}} \geqslant \frac{\omega_{p}^{2}-2 \omega^{2}}{2\left(\omega_{p}^{2}-\omega^{2}\right)}
$$

respectively (Clemmow and Mullaly 1955).

By setting $k=\infty$ in Eq. (1), the resonance cone, $\theta_{\text {res }}$ is obtained. This yields

$$
\tan ^{2} \theta_{\text {res }}=\frac{\left(\omega^{2}-\omega_{p}^{2}-i v \omega !\left[(\omega-i v)^{2}-\omega_{c}^{2}\right]\right.}{\omega_{c}^{2} \omega_{p}^{2}-\left(\omega^{2}-\omega_{p}^{2}-i \omega \omega\right)\left[(\omega-i v)^{2}-\omega_{c}^{2}\right.} .
$$



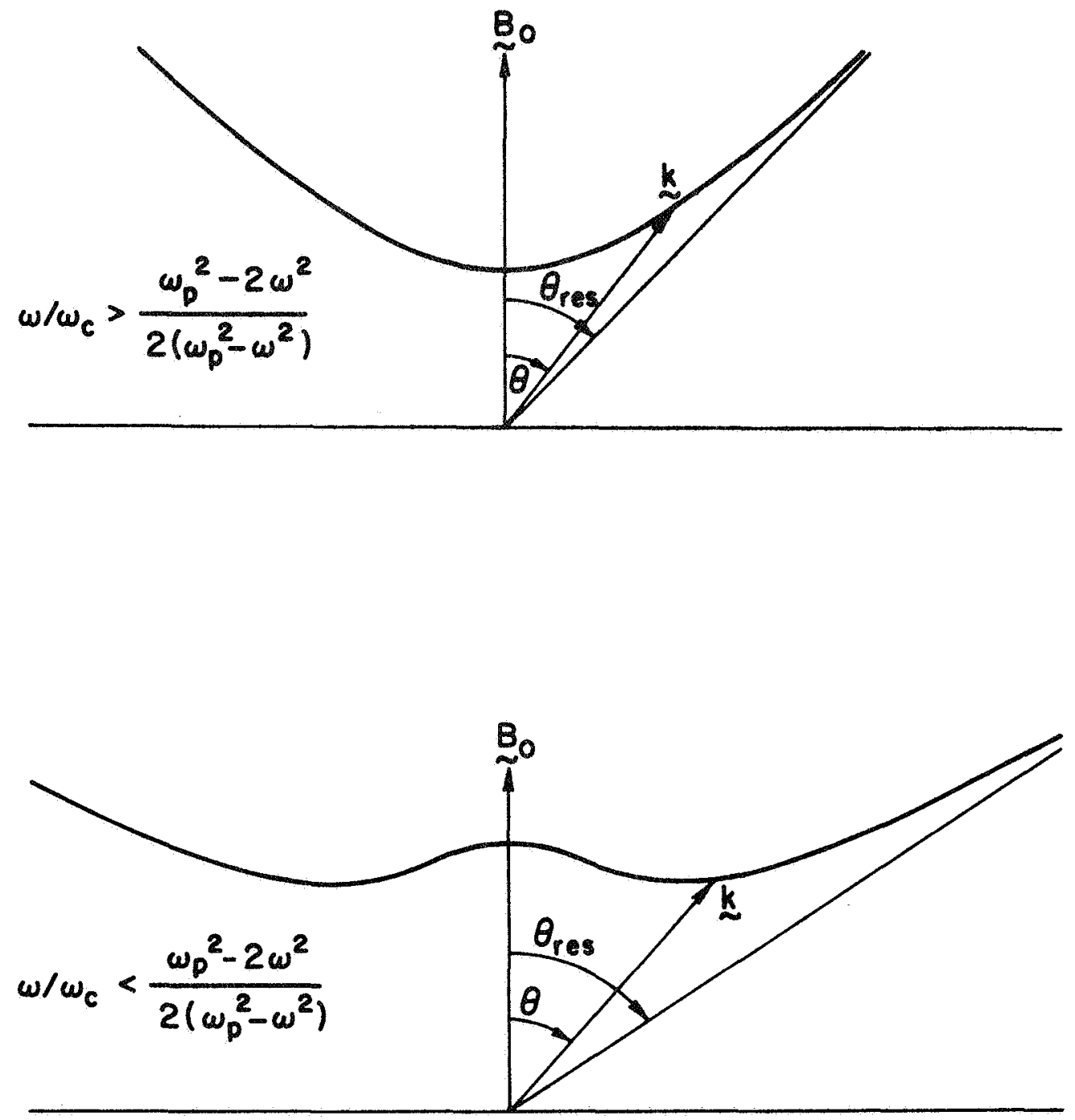

FIG. 2. WHISTLER REFRACTIVE INDEX SURFACES. 
For $v=0$, and under the usual whistler condition $\omega_{p} \gg \omega_{c}>\omega$, the



$$
\theta_{\text {res }}=\tan ^{-1}\left[\frac{\left(w_{p}^{2}-w^{2}\right)\left(w_{c}^{2}-w^{2}\right)}{w^{2}\left(w_{p}^{2}+w_{c}^{2}-w^{2}\right)}\right]^{1 / 2}
$$

Under the quasi-longitudinal condition (Eq. (3)), the resonance angle is given by

$$
\theta_{\text {res }}=\cos ^{-1}\left(\frac{\omega_{c}}{\omega_{c}}\right)^{1 / 2} .
$$

The refractive index surface in Fig. 1 is contained within the resonance cone. The resonance cone is very thin $\left(\theta_{\text {res }} \rightarrow 0\right)$ near cyclotron resonance, and widens as $\omega_{c} / \omega$ increases. For $\omega_{c} / \omega=4.3$, the resonance angle is about $60^{\circ}$. Beyond the resonance angle, the wave is essentially evanescent. Near the resonance cone, the wave will be heavily damped, as can be seen from Eq. (4) for the whistler mode. Typical solutions of Eq. (6) for $v=0$ are shown in Fig. 3.

\subsection{Generalized Snel1's Law}

Consider a plane horizontal boundary between a semi-infinite homogeneous magnetoplasma and a semi-infinite dielectric, as shown in Fig. 4. Let a plane wave be incident obliquely on the boundary from the plasma side, with its wave-normal in the $x-z$ plane at an angle $\theta_{I}$ to the z-axis. Then any field component of this wave is given by

$$
\underset{\Sigma}{\Sigma}={\underset{\sim}{I}}_{I} \exp \left\{-1 \frac{\omega}{c} \mu\left(\theta_{I}\right)\left(z \cos \theta_{I}+x \sin \theta_{I}\right)\right\}
$$

Because of the birefringent nature of the magnetoplasma, in general there will be two reflected waves in the plasma and a transmitted wave in the dielectric, with their wave-normals at angles $\theta_{\mathrm{R} 1}, \theta_{\mathrm{R} 2}$, and $\theta_{\mathrm{T}}$ to the z-axis respectively. The field components, $\underset{\sim}{\mathrm{F}}$ of these waves are given by : 


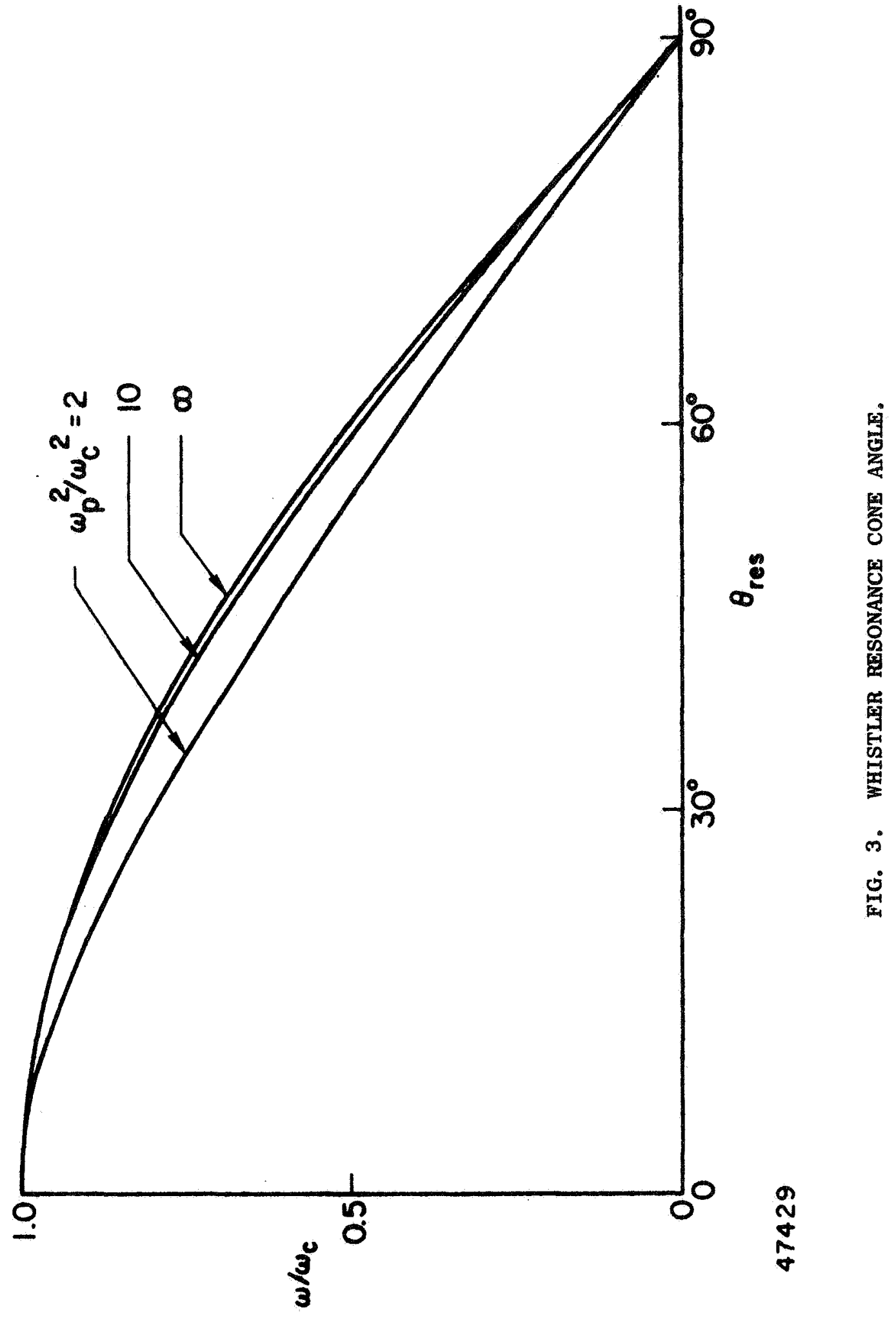




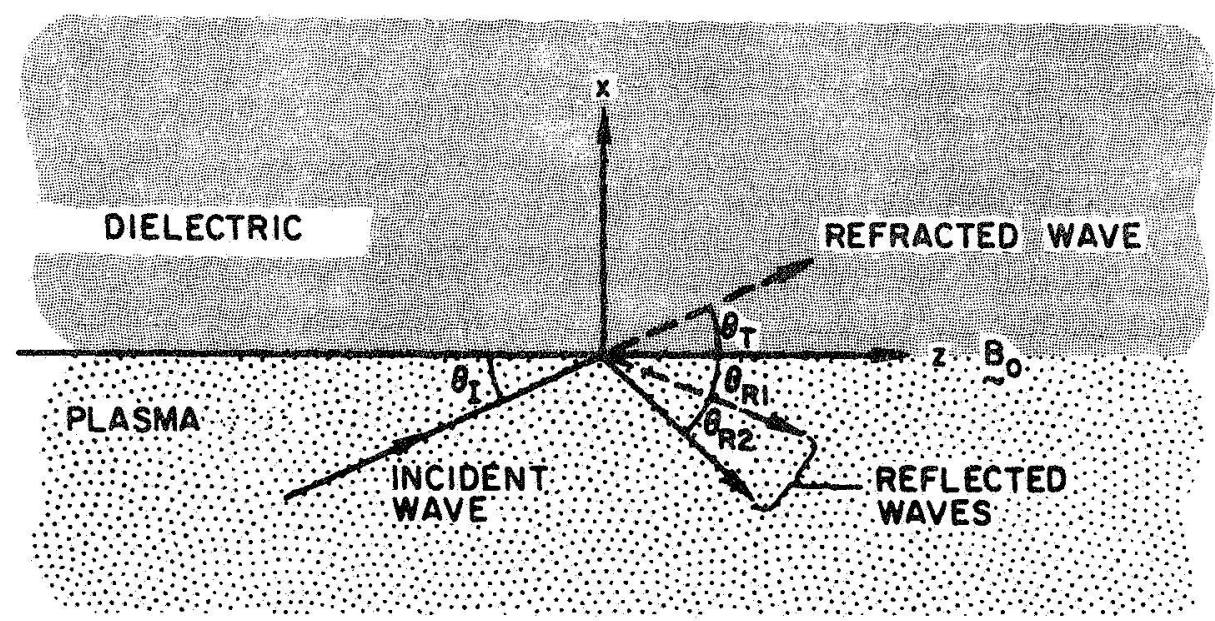

47421

Fig. 4. REFLECTION AND REFRACTION OF A PLANE WAVE AT A SHARP MAGNETOPLASMA BOUNDARY. 
Reflected waves:

$$
\begin{aligned}
& \Sigma_{R}=E_{R I} \exp \left\{-1 \frac{I I}{c} \mu\left(\theta_{R I}\right)\left(z \cos \theta_{R I}-x \sin \theta_{R I}\right)\right\} \\
& +{\underset{\sim}{R 2}} \exp \left\{-1 \frac{\omega}{c} \mu\left(\theta_{R 2}\right)\left(z \cos \theta_{R 2}-x \sin \theta_{R 2}\right)\right\},
\end{aligned}
$$

Transmitted waves:

$$
E_{T}=F_{I} \exp \left\{-1 \frac{\omega}{c} \mu_{d}\left(z \cos \theta_{T}+x \sin \theta_{T}\right)\right\}
$$

where $\mu_{\mathrm{d}}$ is the refractive index of the dielectric.

Since the field components for both sides of the boundary must be in phase over the whole $y-z$ plane, i.e., for $x=0$, we must have

$$
\mu\left(\theta_{I}\right) \cos \theta_{I}=\mu\left(\theta_{R I}\right) \cos \theta_{R I}=\mu\left(\theta_{R 2}\right) \cos \theta_{R 2}=\mu_{d} \cos \theta_{\mathrm{T}} .
$$

Equation (12) is the generalized Sne11's law. By its application, $\theta_{\mathrm{T}}$ can be found easily when $\theta_{I}$ is known. For $\stackrel{B}{\sim}_{0}$ parallel to the z-axis, we can let one of the two reflection angles be equal to $\theta_{I}$ This reflected wave will be called the "principal" reflected wave. For the other reflected wave, which we shall term the "secondary" reflected wave, the Booker quartic is used to find the angle (Budden 1961).

\subsection{The Booker Quartic}

On the plasma side of the boundary, let

$$
S=\mu(\theta) \cos \theta, q=\mu(\theta) \sin \theta .
$$

The quantity $q$, determining the field variations in the $x$-direction, was first introduced into magneto-ionic theory by Booker, and it plays an important part in the theory of radio wave propagation in the ionosphere. The equation governing this quantity depends solely on the properties of the medium, and is, in general, a quartic. When the static magnetic field is parallel to the boundary plane, and in the plane of incidence, the quartic reduces to a biquadratic equation, 


$$
\alpha q^{4}+\gamma q^{2}+\epsilon=0
$$

where

$$
\begin{aligned}
\alpha= & w(w-i v)\left[(w-i v)^{2}-w_{c}^{2}\right]-w_{p}^{2}(w-i v)^{2}, \\
\gamma= & -2(1-i v / w)\left[w(w-i v)-w_{p}^{2}\right]\left[\left(1-s^{2}\right) w(w-i v)-w_{p}^{2}\right] \\
& +2 w_{c}^{2}\left[\left(1-s^{2}\right) w^{2}-w_{p}^{2}\right], \\
\epsilon= & {\left[w(w-i v)-w_{p}^{2}\right]\left[\left(1-s^{2}\right) w(w-i v)-w_{p}^{2}\right] } \\
& -\left(1-s^{2}\right) w_{c}^{2}\left[\left(1-s^{2}\right) w(w-1 v)-w_{p}^{2}\right]-s^{2} w_{p}^{2} w_{c}^{2}\left(1-s^{2}\right) .
\end{aligned}
$$

In a study of waves on an electron beam, Hahn (1939) arrived at the same equation for the separation constants in cylindrical coordinates.

The four solutions of the quartic equation specify four waves. Two solutions represent waves travelling towards the interface; the other two represent waves travelling away from the interface into the plasma. A1l four waves are travelling in the positive z-direction. From Poynting vector considerations, only the latter pair of solutions are of interest.

\subsection{The Reflected and Refracted Fields}

To determine the amplitude and phase of the reflected waves in the plasma, and those of the refracted waves in the isotropic dielectric region, we must match the field components in the plasma side of the interface. With no surface current in the interface, the boundary conditions require the tangential electric field, the normal displacement, and the tangential and normal magnetic fields, to be continuous across the interface. For the incident wave we have: 


$$
\begin{aligned}
& E_{I X}=\cos \theta_{I}+\frac{R\left(\theta_{I}\right) \omega_{c} \omega \sin ^{2} \theta_{I}}{\omega(\omega-i v)-\omega_{p}^{2}}\left[\mu^{2}\left(\theta_{I}\right)-1\right], E_{I y}=1 R\left(\theta_{I}\right), \\
& E_{I z}=\frac{R\left(\theta_{I}\right) \omega_{c} \omega \sin \theta_{I} \cos \theta_{I}}{\omega(\omega-i \nu)-\omega_{p}^{2}}\left[\mu\left(\theta_{I}\right)-1\right]-\sin \theta_{I} \text {, } \\
& H_{I x}=-i \mu\left(\theta_{I}\right) R\left(\theta_{I}\right) \cos \theta_{I}, H_{I y}=\mu\left(\theta_{I}\right), \quad H_{I z}=i \mu\left(\theta_{I}\right) R\left(\theta_{I}\right) \sin \theta_{I}, \\
& R(\theta) \equiv \frac{-\omega_{c} \omega \sin ^{2} \theta}{2\left[\omega(\omega-1 v)-\omega_{p}^{2}\right] \cos \theta}+\left\{\frac{\left(\omega_{c} \omega\right)^{2} \sin ^{4} \theta}{4 \cos ^{2} \theta\left[\omega(\omega-i v)-\omega_{p}^{2}\right]^{2}}+1\right\}^{1 / 2}, \\
& \underset{\sim}{\mathcal{H}} \equiv \underset{\sim}{\mathrm{c}},
\end{aligned}
$$

where $\underset{\sim}{B}$ is the $\mathrm{RF}$ magnetic field. The electric field component perpendicular to the plane wave propagation direction has been normalized to unity.

Let $\Gamma_{1}$ and $\Gamma_{2}$ be the reflection coefficients for the principal and secondary reflected waves in the plasma. The field components for the reflected waves are then given by,

$$
\begin{aligned}
& E_{R x}=\Gamma_{1} E_{I x}+\Gamma_{2}\left[\cos \theta_{R 2}+\frac{R\left(\theta_{R 2}\right) \omega_{c} \omega s^{2} \theta_{R 2}^{2}}{\omega(\omega-1 v)-\omega_{p}^{2}}\left[\mu^{2}\left(\theta_{R 2}\right)-1\right]\right], \\
& \mathbf{E}_{R y}=\Gamma_{1} E_{I y}+1 \Gamma_{2} R\left(\theta_{R 2}\right), \\
& E_{R z}=\Gamma_{1} E_{I y}+\Gamma_{2}\left[\frac{R\left(\theta_{R 2}\right) \omega_{c} \omega \sin \theta_{R 2} \cos \theta_{R 2}}{\omega(\omega-1 v)-\omega_{p}^{2}}\left[\mu^{2}\left(\theta_{R 2}\right)-1\right]-\sin \theta_{R 2}\right],
\end{aligned}
$$




$$
\begin{aligned}
& H_{\mathrm{Rx}}=\Gamma_{1} \boldsymbol{H}_{\mathrm{Ix}}-\Gamma_{2} \mu\left(\theta_{\mathrm{R} 2}\right) \mathrm{R}\left(\theta_{\mathrm{R} 2}\right) \cos \theta_{\mathrm{R} 2}, \boldsymbol{H}_{\mathrm{Ry}}=\Gamma_{1} \boldsymbol{H}_{\mathrm{Iy}}+\Gamma_{2} \mu\left(\theta_{\mathrm{R} 2}\right), \\
& H_{\mathrm{Rz}}=\Gamma_{1} \boldsymbol{K}_{\mathrm{Iz}}+i \Gamma_{2} \mu\left(\theta_{\mathrm{R} 2}\right) \mathrm{R}\left(\theta_{\mathrm{R} 2}\right) \sin \theta_{\mathrm{R} 2} .
\end{aligned}
$$

Because of the circularly polarized nature of the incident wave, we need both E-type (E field in the plane of the incident wave) and H-type (H field in the plane of the incident wave) "refracted" waves, in the isotropic dielectric.

E-wave:

$$
\mathrm{E}_{\mathrm{Tz}}=-\mathrm{T}_{\mathrm{E}} \sin \theta_{\mathrm{T}}, \quad \mathrm{E}_{\mathrm{TX}}=\mathrm{T}_{\mathrm{E}} \cos \theta_{\mathrm{T}}, \quad \boldsymbol{H}_{\mathrm{Ty}}=\mathrm{T}_{\mathrm{E}}
$$

H-wave:

$$
\mathbf{E}_{\mathrm{Ty}}=\mathrm{T}_{\mathrm{H}}, \quad \boldsymbol{H}_{\mathrm{Tx}}=-\mathrm{T}_{\mathrm{H}} \cos \theta_{\mathrm{T}}, \quad \boldsymbol{H}_{\mathrm{Tz}}=\mathrm{T}_{\mathrm{H}} \sin \theta_{\mathrm{T}},
$$

Here, $T_{E}$ and $T_{H}$ are the transmission coefficients. Because of the high refractive index for the incident wave, the waves on the dielectric side are generally evanescent (total reflection). By assuming a rigid boundary, the reflection and transmission coefficients can be determined from the following boundary conditions:

$$
\begin{aligned}
& E_{y}(+0)=E_{y}(-0), \quad E_{z}(+0)=E_{z}(-0), \\
& \mathcal{H}_{y}(+0)=\mathcal{H}_{y}(-0), \quad \mathcal{H}_{z}(+0)=\mathcal{H}_{z}(-0),
\end{aligned}
$$

where the signs " + " and "- refer to the fields immediately adjacent to the boundary on opposite sides of it. These relations are obtained by applying Faraday's law and Ampere's law to a rectangular loop enclosing the boundary. There are two other boundary conditions, namely continuity of normal magnetic field and discontinuity of electric flux density due to surface charge. These are equivalent to Eq. (19) for the boundary considered here. 
When these boundary conditions are applied to Eqs. (15) through (18), we obtain

$$
\begin{aligned}
& i R\left(\theta_{I}\right)+\Gamma_{1} i R\left(\theta_{I}\right)+\Gamma_{2} \operatorname{iR}\left(\theta_{R 2}\right)=T_{H}, \\
& \left(1-\Gamma_{1}\right)\left[\frac{R\left(\theta_{I}\right) w_{c} \omega \sin \theta_{I} \cos \theta_{I}}{\omega(\omega-i \nu)-w_{p}^{2}}\left[\mu^{2}\left(\theta_{I}\right)-1\right]-\sin \theta_{I}\right] \\
& -\Gamma_{2}\left\{\frac{R\left(\theta_{R 2}\right) w_{c} \omega \sin \theta_{R 2} \cos \theta_{R 2}}{w(\omega-i \nu)-w_{p}^{2}}\left[\mu^{2}\left(\theta_{R 2}\right)-1\right]-\sin \theta_{R 2}\right\}=-T_{E} \sin \theta_{T} \text {, } \\
& \mu\left(\theta_{I}\right)+\Gamma_{1} \mu\left(\theta_{I}\right)+\Gamma_{2} \mu\left(\theta_{R 2}\right)=T_{E}, \\
& i \mu\left(\theta_{I}\right) R\left(\theta_{I}\right) \sin \theta_{I}-i \Gamma_{1} \mu\left(\theta_{I}\right) R\left(\theta_{I}\right) \sin \theta_{I} \\
& -i \Gamma_{2} \mu\left(\theta_{R 2}\right) R\left(\theta_{R 2}\right) \sin \theta_{R 2}=T_{H} \sin \theta_{T} \cdot
\end{aligned}
$$

Equation (20) can be solved for $\Gamma_{1}, \Gamma_{2}$ and $T_{H}, T_{E^{*}}$ 


\section{CALCULATIONS}

Starting with $\theta_{I}$, the incident angle, $\mu\left(\theta_{I}\right)$ is calculated from Eq. (1). Then, with $s^{2}=\mu^{2}\left(\theta_{I}\right) \cos ^{2} \theta_{I}$, we determine $q_{1}^{2}$ and $q_{2}^{2}$ from Eq. (14). The two reflection angles are determined by trigonometrical relations in the complex domain. We may assume that the incident wave is a propagating wave $\left(\theta_{I}<\theta_{\text {res }}\right)$, so that the incident angle is real. With this assumption, a typical set of incident and reflected angle relations were calculated for $\theta_{I}=10^{\circ}$, and are shown in Fig. 5 . Note that for $v=0, q_{2}^{2}$ changes sign at $\omega / \omega_{c}=\left(\omega_{p}^{2}-2 \omega^{2}\right) / 2\left(\omega_{p}^{2}-\omega^{2}\right)$, or $\omega / \omega_{c} \approx 1 / 2$ for $\omega_{p}^{2 / \omega^{2}} \gg 1$. This is consistent with the two distinctly different refractive index surfaces shown in Fig. 2.

For whistlers propagating at an angle to the magnetic field within the resonance cone, the refractive index, $\mu_{\left(\theta_{I}\right)}$, is usually very large compared to unity. When $\mu\left(\theta_{I}\right)>\mu_{d}$, the angle of the refracted wave in the dielectric becomes complex and total reflection occurs. The two reflection coefficients are in general complex. A set of typical values for $\Gamma$ calculated from Eq. (20) is given in Fig. 6.

\subsection{Explanation of Wieder's Paradox}

For frequencies above about $\omega_{c^{\prime}} / 2, \quad\left|\Gamma_{1}\right|$ is exactly equal to unity when collisions are neglected, and $\left|\Gamma_{2}\right|$ is of order of unity. Since, then, the secondary reflected wave has very large imaginary transverse propagation constants, it is localized at the boundary. The secondary reflected wave is a whistler "propagating" at an imaginary angle. Another boundary placed more than a few skin-depths away (say 10), will not change the secondary reflected fields appreciably. Thus the primary reflected wave will be reflected back and forth between the boundaries, just as in an ordinary dielectric slab waveguide. This is illustrated in Fig. 7.

Below about half the cyclotron frequency, the secondary reflected wave also propagates as a whistler, propagating at a larger real angle than the principal reflected wave. If another boundary is present, the effect of the secondary reflected wave cannot be neglected, no matter how far away the other boundary is located. 


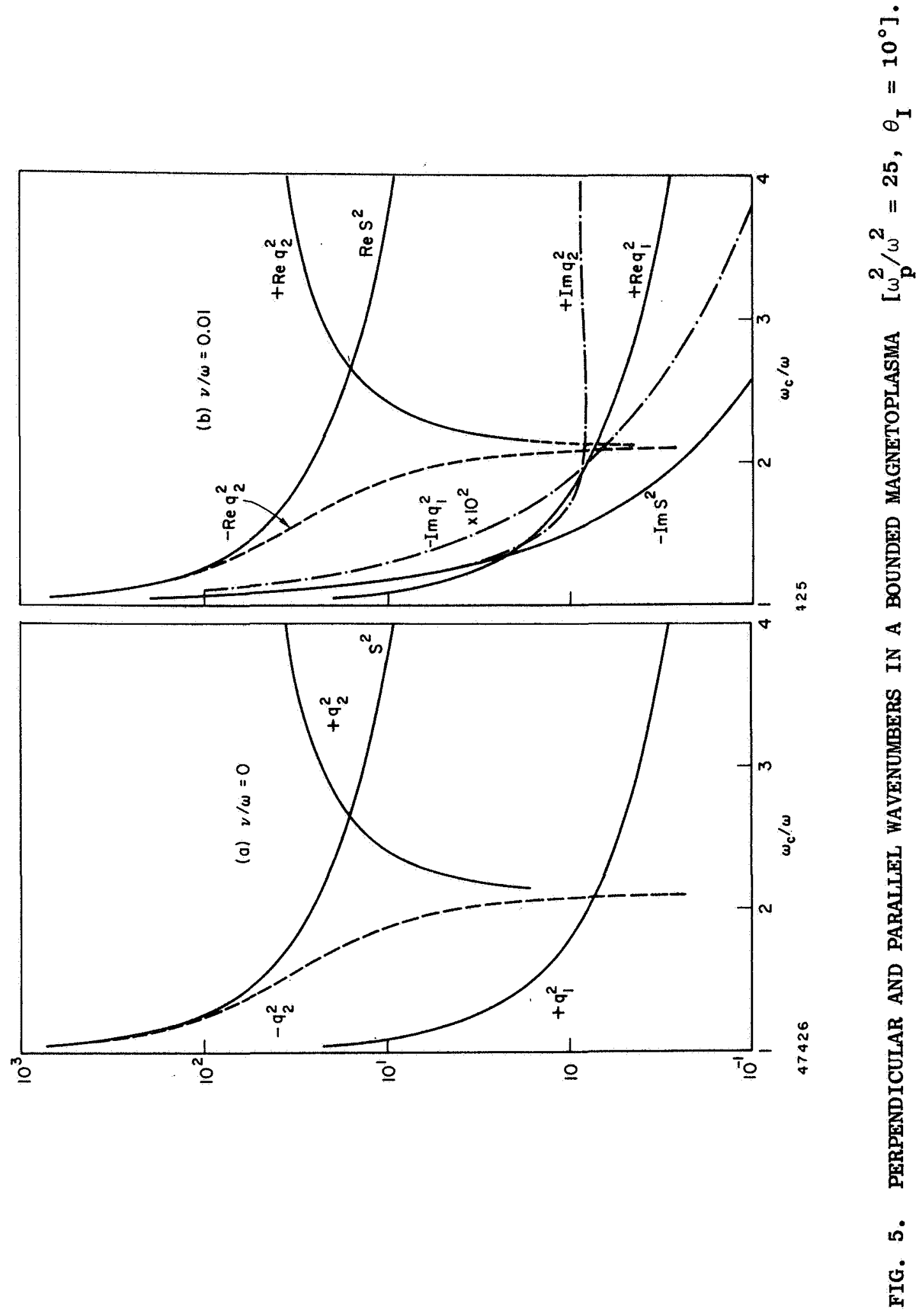



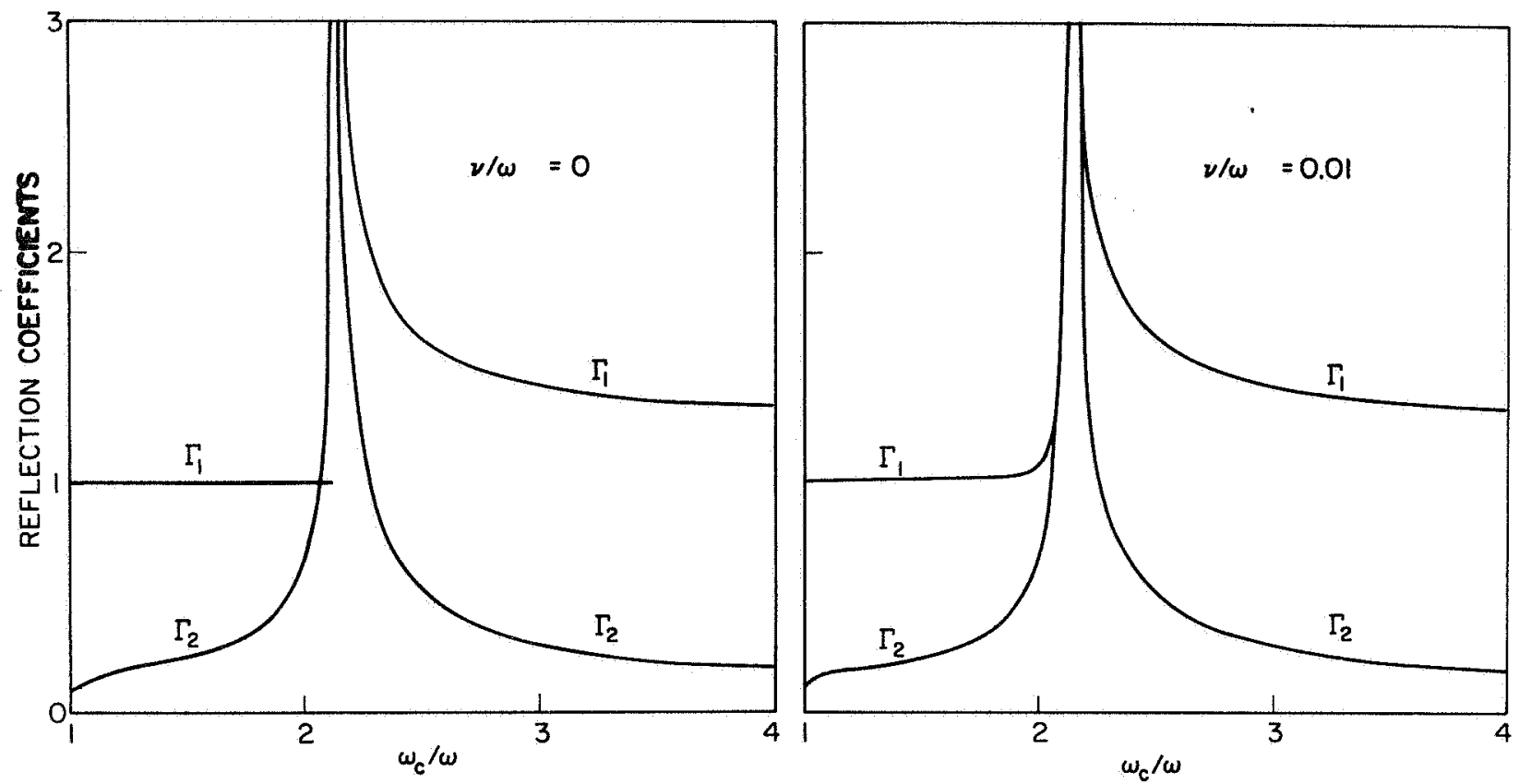

47433

(a) VACUUM - PLASMA INTERFACE
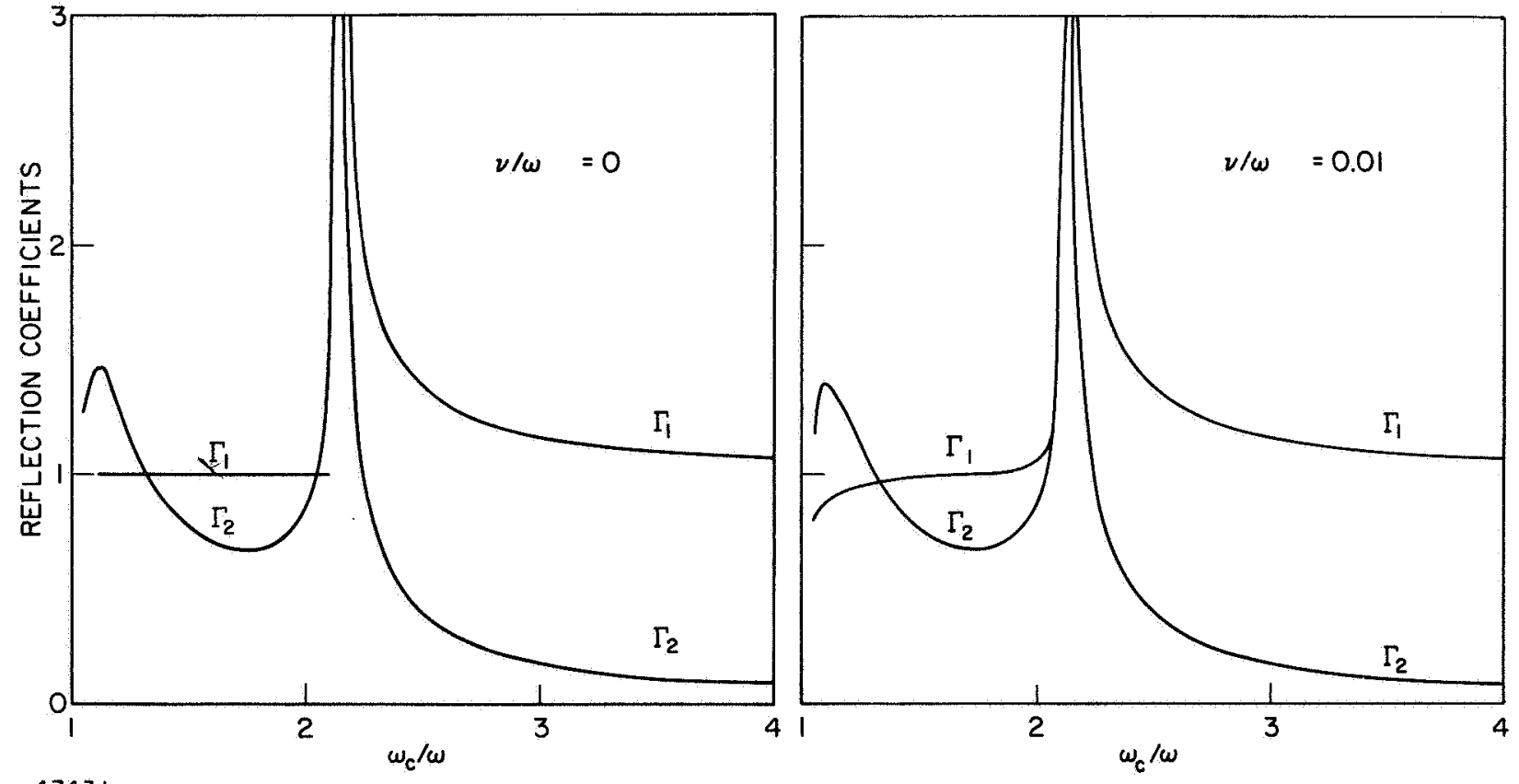

47431

(b) CONDUCTOR - PLASMA INTERFACE

Fig. 6. PRINCIPAL AND SECONDARY REFLECTION COEFFICIENTS FOR WHISTLER INCIDENT AT AN ANGLE TO A SHARP MAGNETOPLASMA BOUNDARY $\left[\omega_{p}^{2} / \omega^{2}=25, \theta_{I}=10^{\circ}\right]$. 

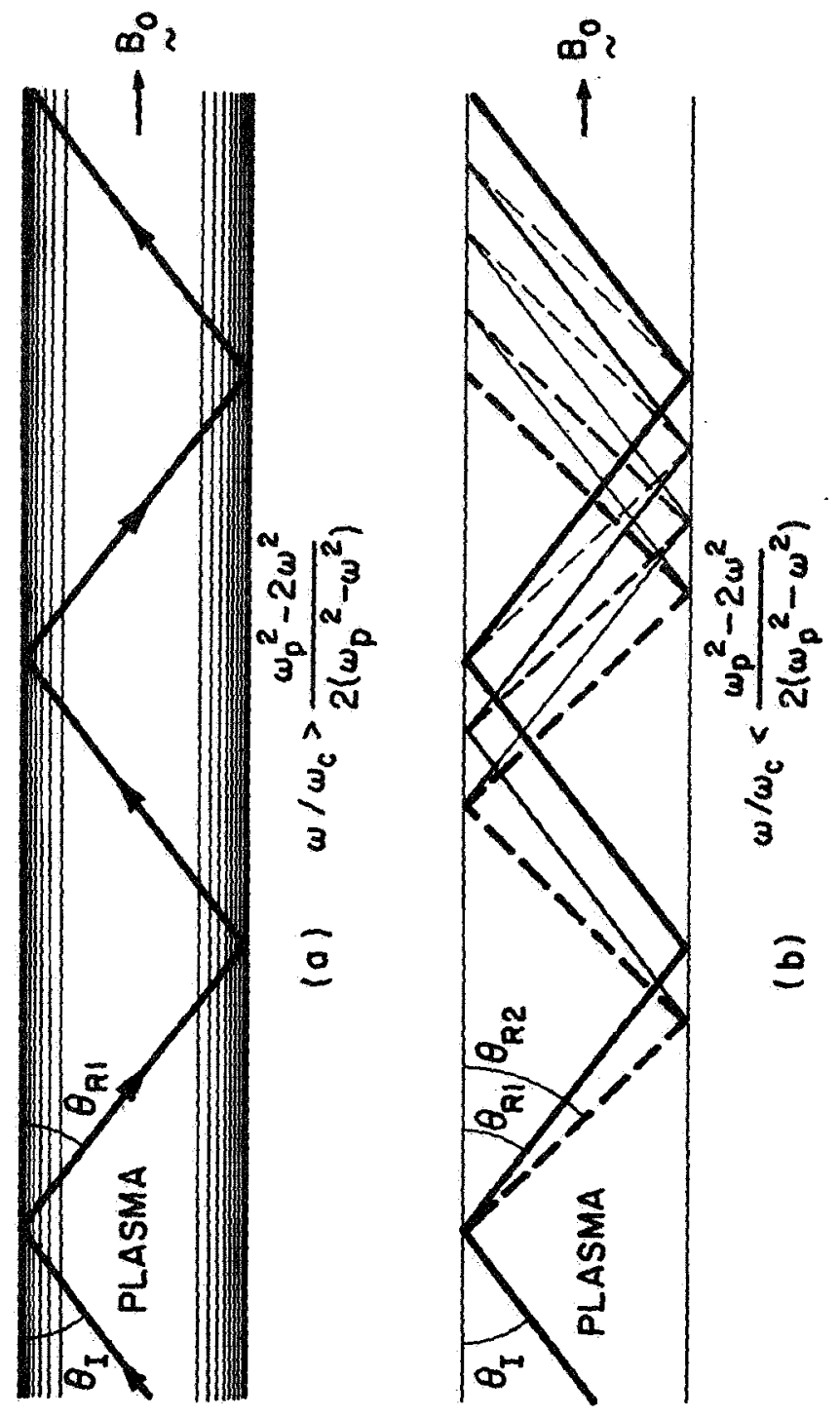

垈 
Since in each reflection a new propagating wave is born, as shown in Fig. 7 (b), a spectrum of waves finally results. This explains the interthe interference pattern manifested in the calculations of ck/ $\omega$ by Wieder (1964). A few of his results are shown in Fig. 8 (a). If collisions or electron temperature are introduced into the plasma mode1, we see in Fig. 2 that for small angles of incidence (grazing incidence), the primary reflected wave will be far away from the resonance cone, and will suffer little damping. Since the secondary reflected wave propagates at an angle much closer to the resonance cone, it will suffer much heavier damping, as seen from Eq. (4). Thus, with enough collisions, the interference pattern should disappear. Furthermore, as the wavelength is made small compared with the width of the plasma slab, e.g., by increasing $\omega_{p}$, the plane wave solution for an unbounded plasma should be approached. This has been found by Hedvall and Sjogren. One of their results for ck/ $\omega$ is shown in Fig. 8 (b).

\subsection{Limitations of the Quasistatic Approximation}

A number of authors (Scharer and Trivelpiece 1965, Bevc 1967) have attempted to establish a general criterion for validity of the quasistatic approximation. By comparing a limited set of numerical calculations with the full Maxwell equations, and with the quasistatic approximation, it seemed that $\omega_{p} a / c \ll 1$ could be used as the criterion to justify the quasistatic approximation (Gould 1961), where a is the plasma column radius. Based on a quasistatic analysis of power flow, additional criteria for the extension of the quasistatic approximation were derived (Scharer and Trivelpiece 1965). However, Bevc (1966, 1967) claims that quasistatic calculations of power flow are unsatisfactory, and proposes to compare the laminar and solenoidal parts of the RF electric field as a criterion. It is true that in general

$$
\underset{\sim}{\mathbf{E}}=-\nabla \psi-\mathbf{i} \omega \underset{\sim}{A},
$$

and that when $|\nabla \psi| /|\omega \mathrm{A}| \gg 1$, the quasistatic approximation is justified. However, $\underset{\sim}{\mathrm{A}}$ and $\psi$ are coupled, and the comparison can only be made after the full Maxwell equations are solved. This is not satisfactory, because the advantage of the quasistatic approximation has been to avoid using the ful1 Maxwel1 equations. 


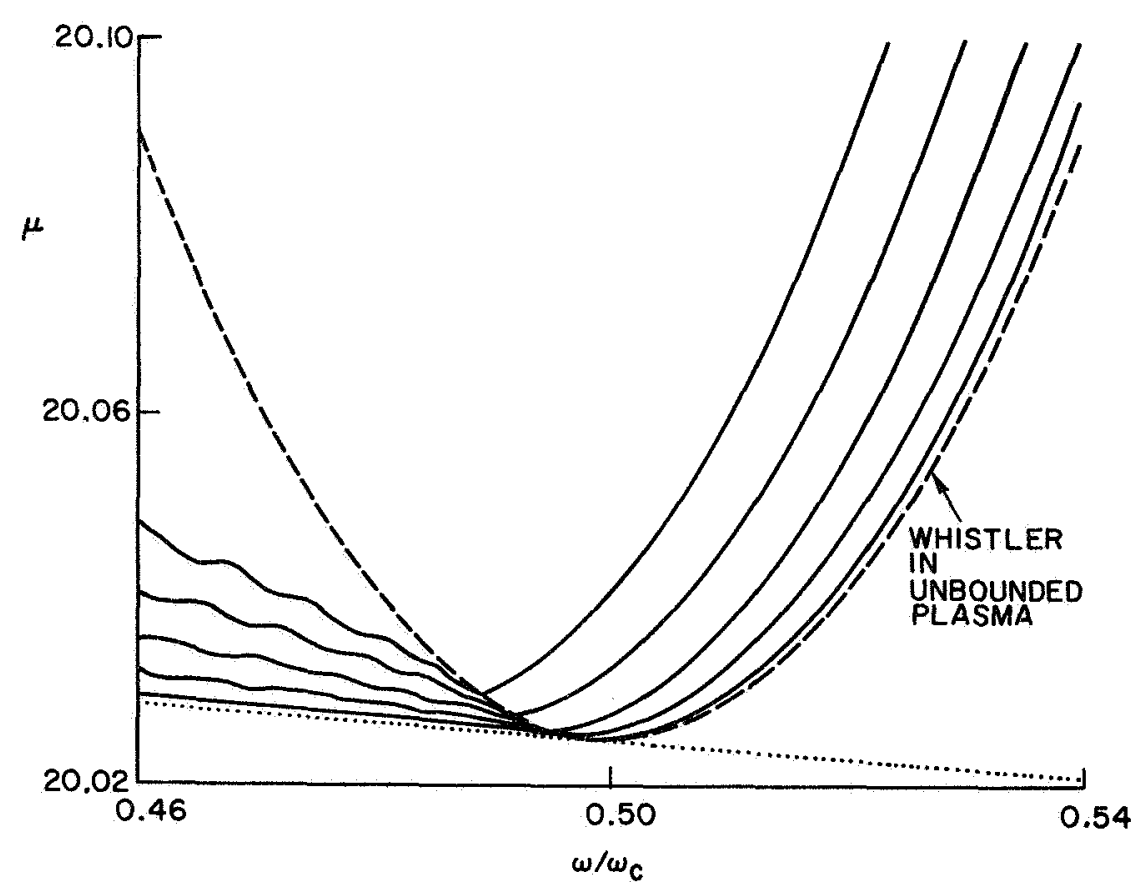

(a) Wieder's results [circular waveguide filled with magnetoplasma, $\omega_{p}^{2} / \omega_{c}^{2}=100, \quad a \omega_{c} / c=\pi, \quad a$ is plasma radius $]$.

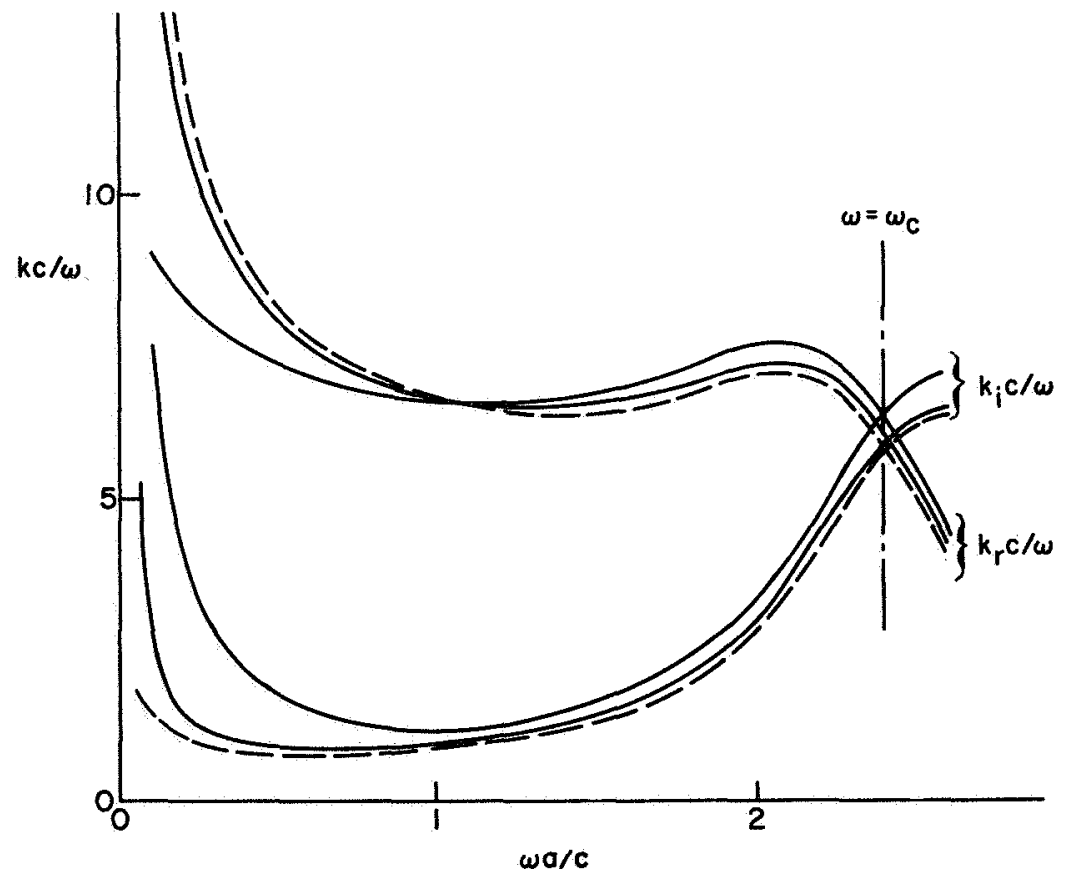

(b) Hedvall and Sjogren's results [plasma slab in vacuum, $\omega_{\mathrm{p}}^{2} / \omega_{\mathrm{c}}^{2}=11$, $a \omega_{c} / c=2.4, \quad a$ is plasma width].

FIG. 8. WHISTLER DISPERSION CHARACTERISTICS IN BOUNDED MAGNETOPLASMA. 
For a waveguide completely filled with plasma in a uniform magnetic field, there are two groups of waves with strong field inside the plasma. These waves will be called body waves. For $\omega_{p}>\omega_{c}$, one group of backward waves propagate in the band between the hybrid frequency, $\omega_{h}\left[=\left(\omega_{p}^{2}+\omega_{c}^{2}\right)^{1 / 2}\right]$, and the plasma frequency. Another group of slow waves propagate from the cyclotron frequency down to zero frequency. The forward slow wave of the quasistatic approximation can be viewed as a whistler propagating very close to the resonance cone and reflected at the boundary. Whistlers propagating at an angle to the external magnetic field, ${ }_{\sim}^{\mathrm{B}}$, are physically coupled to plasma oscillations. Close to the resonance cone, the axial electric field dominates over the RF magnetic field. Therefore, the RF magnetic field can be neglected. From Eq. (15), the ratio of $\mathrm{RF}$ electric field, $E$, to RF magnetic field, $\mathcal{H}$, in a collisionless magnetoplasma, close to the resonance cone, is given by

$$
\frac{|E|}{|\mathcal{H}|}=\frac{\omega_{c} \omega \sin \theta}{\left|\omega^{2}-\omega_{p}^{2}\right|} \mu(\theta)=\frac{\omega_{c} \omega \sin \theta \lambda_{0}}{\left|\omega^{2}-\omega_{p}^{2}\right| \lambda(\theta)},
$$

where $\lambda_{0}$ is the signal wavelength in vacuum, and $\lambda$ is the signal wavelength in the plasma. The width of the plasma slab, a, can be expressed in terms of the angle of propagation by the familiar waveguide relation

$$
a=\frac{m \lambda(\theta)}{2 \sin \theta}=\frac{m \lambda_{0}}{2 \sin \theta \mu(\theta)},
$$

where $m$ is the transverse mode number. For bounded magnetoplasma, due to the secondary reflected wave, $m$ is in general a fractional number. When $\theta$ is exactly equal to $\theta_{\text {res' }}$ the refractive index, $\mu$, is infinite, and the size of the waveguide would have to be zero unless $\theta$ is also zero. But, in general, $\theta_{\text {res }} \neq 0$. Thus, in order to obtain a predominantly electrostatic solution, we must make $\theta$ close to $\theta_{\text {res }}$ so that the refractive index becomes very large but remains finite. Combining Eqs. (22) and (23), for $|E| \gg|\boldsymbol{H}|$ we have 


$$
\frac{m \pi w_{c} \omega_{c}}{\left|w^{2}-w_{p}^{2}\right|^{a}} \gg 1 .
$$

This is the condition for the validity of the quasistatic approximation. The condition given by Eq. (24) can be applied to both the forward and backward body wave modes. For the forward wave modes under the usual whistler conditions, $\omega_{p} \gg \omega_{c}>\omega$, Eq. (24) reduces to

$$
\frac{m \pi \omega_{c}}{\omega_{p}^{2}} \gg 1
$$

For the quasistatic approximation to be valid, we need strong magnetic fields, and/or low plasma densities and small plasma column cross-sections. The approximation is also improved by increasing the mode number. All these features agree with the results of numerical calculations shown in Fig. 9 (Gould 1961). 


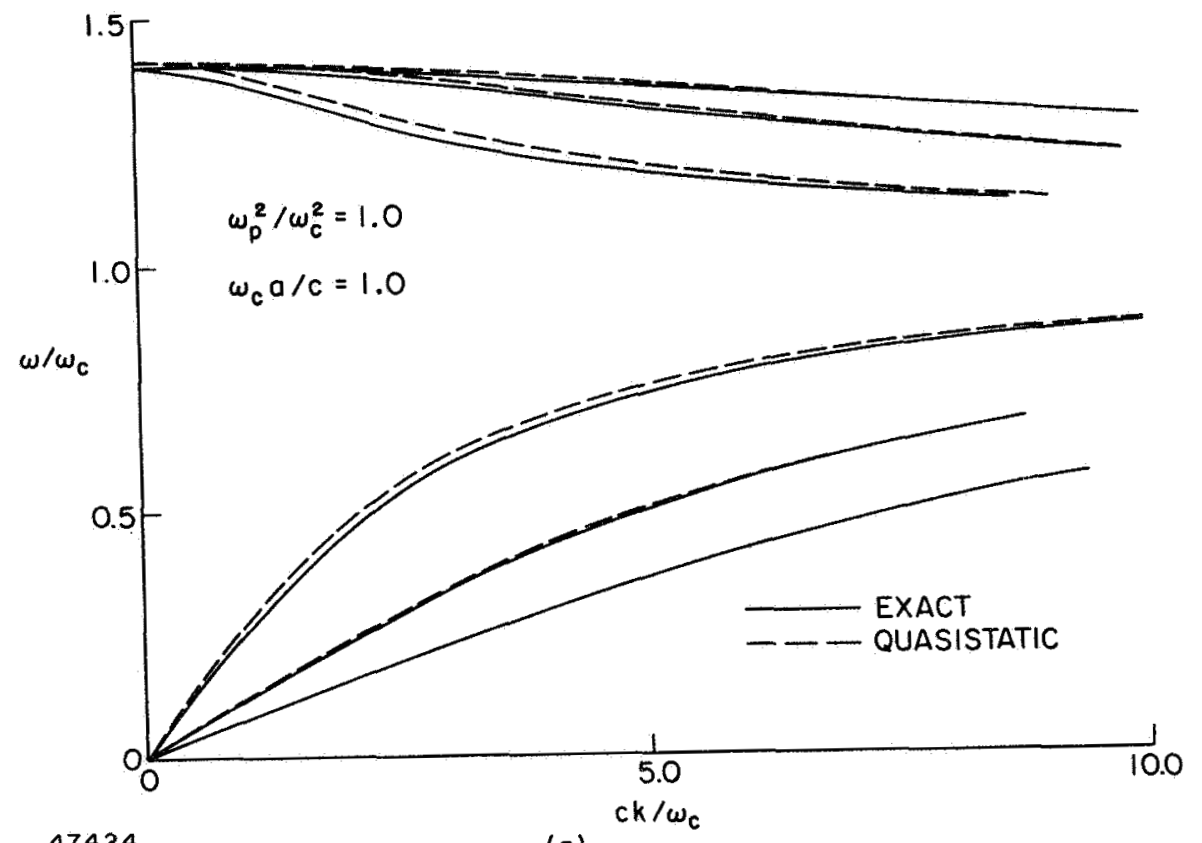

47424

(a)

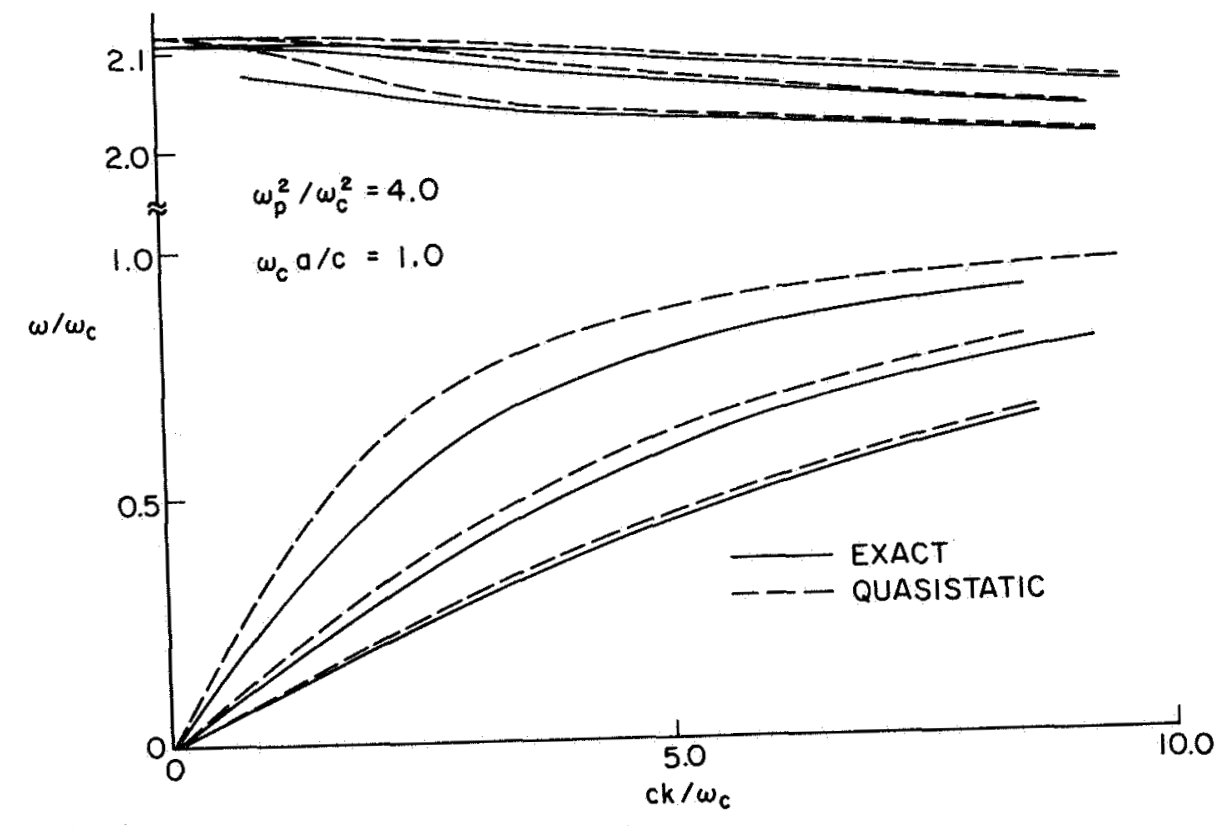

47423

(b)

FIG. 9. COMPARISON OF BODY WAVE DISPERSION CURVES IN MAGNETOPLASMA FILLED WAVEGUIDE CALCULATED FROM FULL MAXWELL EQUATIONS, AND WITH QUASISTATIC APPROXIMATION (Gould 1961). 


\section{DISCUSSION}

In the preceding sections, we have explained Wieder's paradox as an interference pattern of plane waves which double due to double reflection. Our analysis also connects the well-studied plane wave theory in unbounded plasmas directly with the theory of waves in bounded plasmas. Since cylindrical waves and spherical waves can be synthesized in terms of plane waves (Stratton 1941), our result applies also to cylindrical and spherical configurations. This approach can be used for other modes, e.g., the backward wave which should propagate in a bounded magnetoplasma in the frequency range $\omega_{h}>\omega>\omega_{p}$ for $\omega_{p}>\omega_{c}$. This mode can be related to the extraordinary wave in Region 5 of Fig. 1.

From the point of view of plane wave reflections, the limitations of the quasistatic approximation for body waves in bounded magnetoplasma has been established. For a waveguide partially filled with plasma, surface wave modes may propagate. Since the energy in surface waves is concentrated near the plasma boundary, the above conditions for the validity of the quasistatic approximation cannot be applied to them. For surface waves, the usual slow wave criterion is generally adequate.

\section{ACKNOWLEDGEMENTS}

The author would like to thank Drs. F. W. Crawford, H. Derfler and R. A. Helliwell for valuable comments on this work. 
Allis, W. P., Buchsbaum, S. J., and Bers, A., 1963, Waves in Anisotropic Plasmas (MIT Press, Cambridge, Mass).

Bevc, V., 1966, J. Appl. Phys. 37, 3128; 1967, Proc. Eighth International Conference on Phenomena in Ionized Gases, Vienna (International Atomic Energy Agency), p.333.

Budden, K. G., 1961, Radio Waves in the Ionosphere, (Cambridge Univ. Press, London).

Clemmow, P. C., and Mullaly, R. F., The Physics of the Ionosphere (Physical Society, London) p. 340 .

Formato, D., and Gilardini, A., 1962, J. Res. NBS 66D, 543.

Gould, R. W., 1961, California Institute of Technology, Contract DA36-039SC-85317 Final Report (unpublished).

Hahn, W. C., 1939, General Electric Rev. 42, 258.

Hedval1, P., and Sjogren, 1965, Elect. Letters 1, 54.

Helliwe11, R. A., 1965, Whistlers and Related Ionospheric Phenomena

(Stanford, Calif.)

Scharer, J. E., and A. W. Trivelpiece, 1965, J. Appl. Phys. 36, 318.

Smuliin, L. D., and Chorney, P., 1958, Proc. Symposium on Electronic Waveguides (Brooklyn Polytechnic Press, Brooklyn, N.Y.) p. 229.

Stratton, J. A., 1941, Electromagnetic Theory, (McGraw-Hill Book Company, New York).

Trivelpiece, A. W., and Gould, R. W., 1959, J. App1. Phys. 30, 1784. Wieder, B., 1964, Phys. Fluids $\underline{7}, 964$. 